\title{
6. Criterios histopatológicos para el diagnóstico de fibrosis pulmonar idiopática
}

\author{
CRISTINA FERNÁNDEZ F** y MANUEL MENESES C.**
}

\section{Histopathological criteria for the diagnosis of idiopathic pulmonary fibrosis}

In the latest update of the ATS/ERS/JRS/ALAT Clinical Practice Guidelines for idiopathic pulmonary fibrosis (IPF), a new way of classifying histopathological patterns in 4 types is proposed: definitive usual interstitial pneumonia (UIP), probable UIP, indeterminate and alternative to UIP. A heterogeneous fibrotic remodeling of the normal architecture of the pulmonary parenchyma, with destructive scarring in the form of "honeycomb", presence of fibroblastic foci and predominantly subpleural and paraseptal distribution, with scarce chronic interstitial inflammatory infiltrate, associated with the absence of elements suggestive of secondary causes such as bronchiolocentric distribution, predominance of inflammatory interstitial infiltrates or poorly formed granulomas, allows an accurate diagnosis of IPF in an appropriate clinical-radiological scenario.

Key words: Idiopathic pulmonary fibrosis; Fibroblasts; Architecture.

\section{Resumen}

En la última actualización de las Guías de Práctica Clínica de la ATS/ERS/JRS/ALAT de fibrosis pulmonar idiopática (FPI) se propone una nueva forma de clasificar los patrones histopatológicos en 4 tipos: definitivo de neumonía intersticial usual (NIU), probable NIU, indeterminado y alternativo a NIU. Una remodelación fibrótica heterogénea de la arquitectura normal del parénquima pulmonar, con cicatrización destructiva en forma de "panal de abejas", presencia de focos fibroblásticos y distribución predominantemente subpleural y paraseptal, con escaso infiltrado inflamatorio intersticial de tipo crónico, asociado a la ausencia de elementos sugerentes de causas secundarias como distribución bronquiolocéntrica, predominio de infiltrados intersticiales inflamatorios o granulomas mal formados, permite un diagnóstico certero de FPI en un escenario clínico-radiológico adecuado.

Palabras clave: Fibrosis pulmonar idiopática; Fibroblastos; Arquitectura.

En la última actualización de las Guías de Práctica Clínica de la ATS/ERS/JRS/ALAT de FPI se propone una nueva forma de clasificar los patrones histopatológicos, la que define 4 tipos:

\section{El patrón definitorio de neumonía intersticial usual (NIU) incluye:}

- Una remodelación fibrótica de la arquitectura normal del parénquima pulmonar, con cicatrización destructiva en forma de "panal de abejas", con metaplasia de musculatura lisa (Figura 1).
- De distribución predominantemente subpleural y paraseptal.

- Compromiso heterogéneo de las lesiones (alternancia de tejido de cicatriz con parénquima relativamente preservado, que se aprecia con aumento menor).

- Puede haber infiltrado inflamatorio intersticial de tipo crónico escaso, no relevante, en áreas linfoplasmocitoide, que se asocian a hiperplasia de pneumocitos tipo II.

- En el frontis de la fibrosis presencia de focos fibroblásticos.

- Ausencia de hallazgos sugerentes de diagnósticos alternativos.

\footnotetext{
* Instituto Nacional del Tórax. Profesor asistente Universidad de Chile. Santiago, Chile.

** Instituto Nacional del Tórax. Santiago, Chile.
} 


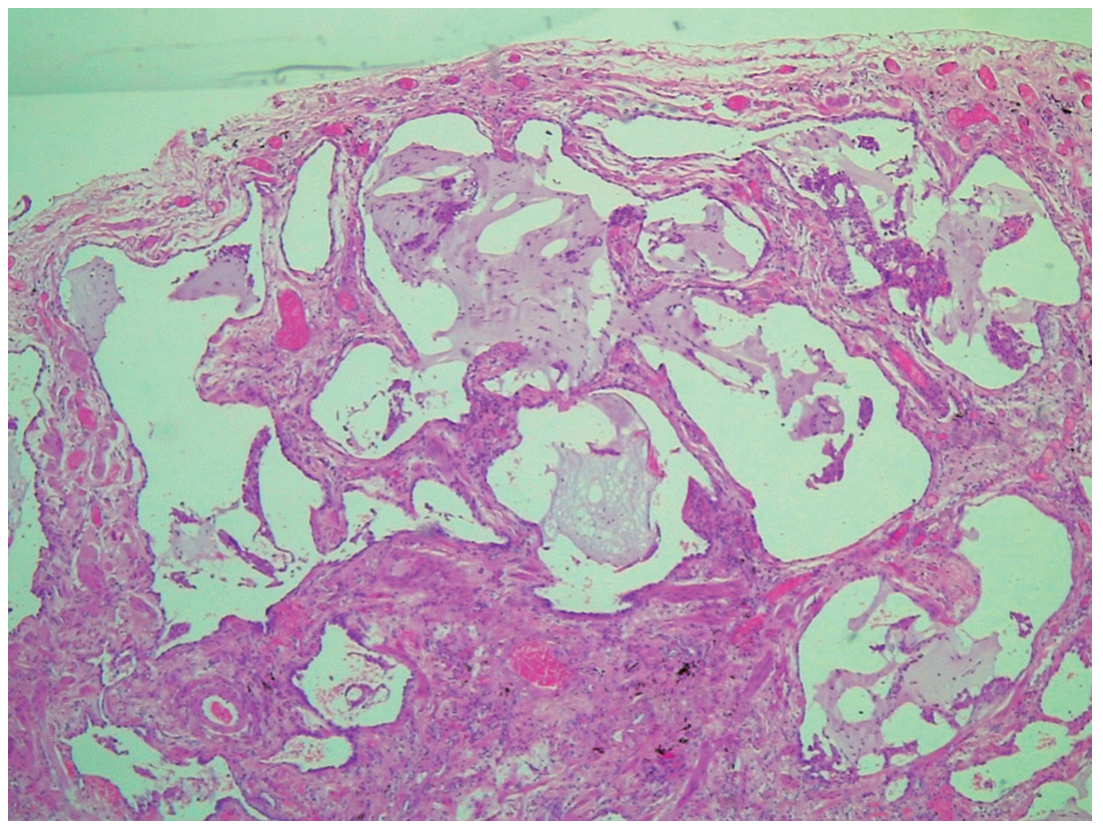

Figura 1. Fibrosis subpleural con panal, sugerente de patrón NIU definitivo.

\section{El patrón probable neumonia intersticial usual (NIU)}

- Muestra algunos hallazgos de NIU pero de menor extensión o profusión, sin hallazgos sugerentes de diagnósticos alternativos; o bien, muestra la presencia de panal de abeja aislado.

\section{El patrón indeterminado}

- Es aquel que muestra un patrón fibrótico diferente a NIU (granulomas, membranas hialinas, cambios centrados en la vía aérea predominantemente, áreas de inflamación intersticial sin fibrosis asociada, pleuritis fibrótica crónica marcada, neumonía en organización).

- NIU pero de causa secundaria (por ej. enfermedades del tejido conectivo o neumonitis por hipersensibilidad):

- Inflitrado inflamatorio celular alejado de las áreas de panal de abeja, hiperplasia linfoide prominente incluyendo centros germinales secundarios (más de 3 en un campo de aumento menor), recuento de células plasmáticas $>40$ en campo de aumento mayor.

- Ausencia de focos fibroblásticos.

- Distribución bronquiolocéntrica evidente, incluyendo metaplasia peribronquiolar extensa, fibrosis en puentes (de bronquiolo a bronquiolo) y/o metaplasia bronquiolar y/o focos fibroblásticos en relación a la vía aérea.

\section{Patrón sugerente de diagnóstico alternativo}

Como otras neumonías intersticiales idiopáticas u otras EPI como neumonitis por hipersensibilidad, histocitosis de células de Langerhans, sarcoidosis, linfangioleiomiomatosis, etc.

Es importante establecer parámetros de calidad respecto a la obtención de la muestra:

- Dos sitios diferentes de biopsia con muestras de no menos de $3,5 \mathrm{~cm} \times 2 \mathrm{~cm}$ con corte de la sutura mecánica y fijación en formalina tamponada al $10 \%$ por unas 6 o más horas y menos de 72 .

- Las muestras se deben fijar desprovistas de sutura mecánica.

- Se debe evitar artefacto de atelectasia, idealmente retirar la sutura antes de introducir en formalina y, una vez cerrado con tapa rosca, agitar para expandir el parénquima pulmonar.

- Cada fragmento de pulmón se debe cortar para dejar una superficie pleural para evaluación.

- Es más elegible biopsia de región media y en la base del pulmón. Se deben evitar las zonas de peor aspecto, con fibrosis terminal.

- Las muestras pueden ser obtenidas por videotoracoscopía (VATS) o cirugía abierta.

- Ocasionalmente el material puede ser obtenido de resecciones segmentarias, lobectomía o resecciones atípicas de tumor pulmonar $u$ otra cirugía diagnóstica torácica que permita evaluar pulmón. 
- Biopsia transbronquial: las muestras de biopsia transbronquial pueden tener elementos que permitan plantear un diagnóstico alternativo, sin ser éste, de ningún modo, definitivo.

\section{Bibliografía}

1.- RAGHU G, REMY-JARDIN M, MYERS JL, RICHELDI L, RYERSON CJ, LEDERER DJ, et al. Diagnosis of idiopathic pulmonary fibrosis: an official
ATS/ERS/JRS/ALAT clinical practice guideline. Am J Respir Crit Care Med. 2018;198:e44-e68.

2.- QURESHI RA, AHMED TA, GRAYSON AD, SOORAE AS, DRAKELEY MJ, PAGERD. Does lung biopsy help patients with interstitial lung disease? Eur J Cardiothorac Surg 2002; 21: 621-6.

3.- KHALIL M, COWEN M, CHAUDHRY M, LOUBANI M. Single versus multiple lung biopsies for suspected interstitial lung disease. Asian Cardiovasc Thorac Ann 2016; 24: 788-91. 